\title{
AUTOMORPHISM GROUPS OF JORDAN ALGEBRAS
}

\author{
E. RAY BOBO
}

Introduction and the Jordan algebras. In his development of a structure theory for Jordan algebras of characteristic two, E.C. Paige [1] introduces an important class of central simple Jordan algebras $S\left[2^{n}\right]$. It is the purpose of this paper to completely determine the automorphism groups of the algebras $S\left[2^{n}\right]$. The automorphisms will be represented as matrices operating on a natural basis for the underlying vector space of the algebra. Using this characterization, generators and relations will be obtained for each of the automorphism groups. In this way, we will produce an infinite family of finite 2-groups.

Definition. Let $S\left[2^{n}\right]$ be the vector space over the field $J_{2}$ of two elements with basis $\left\{u_{-1}, u_{0}, u_{1}, \cdots, u_{m}\right\}$ where $m=2^{n}-2$ for $n \geq 2$. Let

$$
\begin{aligned}
& u_{0} u_{i}=u_{i} \text { for }-1 \leq i \leq 2^{n}-2, \\
& u_{-1}^{2}=0, \\
& u_{j} u_{-1}=u_{j-1} \text { for } 0 \leq j \leq 2^{n}-2, \\
& u_{i} u_{j}=u_{j} u_{i} \text { for }-1 \leq i, j \leq 2^{n}-2 .
\end{aligned}
$$

For $n=2$, set $u_{1} u_{2}=u_{1}^{2}=u_{2}{ }^{2}=0$. For $k>2$, consider $S\left[2^{k}\right]$ as a subspace of $S\left[2^{k+1}\right]$. Then define

$$
u_{r+i} u_{j}=g_{i, j} u_{r+i+j}
$$

for $r=2^{k},-1 \leq i \leq r-2$, and $0 \leq j \leq r-2$ when $u_{i} u_{j}=g_{i, j} u_{i+j}$ where $g_{i, j}$ is 0 or 1 . Also define

$$
u_{r+i} u_{r+j}=0
$$

for $r=2^{k}$ and $-1 \leq i, j \leq r-2$.

\section{The automorphisms.}

Definition. Define a set of $2^{n}$ by $2^{n}$ matrix forms $A_{n}$ inductively as follows: Let

Received April 18, 1967.

This paper represents a portion of the author's doctoral dissertation written at the University of Virginia under the direction of Professor Eugene C. Paige. 


$$
A_{2}=\left(\begin{array}{cccc}
1 & 0 & a_{1} & a_{2} \\
0 & 1 & 0 & 0 \\
0 & 0 & 1 & 0 \\
0 & 0 & 0 & 1
\end{array}\right)
$$

where $a_{1}$ and $a_{2}$ are elements of the field $J_{2}$. Set $r=2^{k}, s=2^{k-1}$, and $t=2^{k+1}$. Assume that $A_{k}=\left(b_{i, j}\right)$ is defined in terms of elements $a_{e}$ of $J_{2}$ where $1 \leq e \leq r-2$ and $-1 \leq i, j \leq r-2$. Then define $A_{k+1}=\left(c_{i, j}\right)$ in terms of elements $a_{e}$ of $J_{2}$ where $1 \leq e \leq t-2$ and $-1 \leq i, j \leq t-2$ by

$$
\left\{\begin{array}{l}
c_{i, j}=b_{i, j} \\
c_{r+i, r+j}=b_{i, j} \\
c_{r+i, j}=0
\end{array}\right.
$$

for $-1 \leq i, j \leq r-2$, and

$$
\left\{\begin{array}{l}
c_{i, r+j}=h_{i, s+j} a_{s+e} \\
c_{i, r+s+j}=h_{i, s+j} a_{r+e} \\
c_{s+i, r+s+j}=h_{i, s+j} a_{s+e} \\
c_{s+i, r+j}=0
\end{array}\right.
$$

for $-1 \leq i, j \leq s-2$ and $1 \leq e \leq r-2$ when $b_{i, s+j}=h_{i, s+j} a_{e}$ where $h_{i, s+j}$ is 0 or 1 .

Theorem 1. The matrices of form $A_{n}$ are precisely the matrices of the automorphisms of the Jordan algebra $S\left[2^{n}\right]$ over the field $J_{2}$ with respect to the canonical basis.

Proof. The proof is by induction on $n$. It is straightforward to show that the theorem is true for $n=2$.

For the remainder of this paper, set $r=2^{k}, s=2^{k-1}$, and $t=2^{k+1}$. Let $\mathscr{A}_{n}$ be the set of all automorphisms of $S\left[2^{n}\right]$ over $J_{2}$. Suppose that the matrices of form $A_{k}$ are precisely the matrices of the elements of $\mathscr{A}_{k}$ with respect to the canonical basis elements $u_{i}$ where $-1 \leq i \leq r-2$. We will show that the matrices of form $A_{k+1}$ are precisely the matrices of the elements of $\mathscr{A}_{k+1}$ with respect to the canonical basis elements $u_{i}$ where $-1 \leq i \leq t-2$.

Let $C \in \mathscr{A}_{k+1}$ and denote 


$$
C\left(u_{i}\right)=\sum_{e=-1}^{t-2} c_{i, e} u_{e} \text { for }-1 \leq i \leq t-2 .
$$

Let $\delta_{i, j}$ denote the Kronecker delta. It is fairly easy to verify each of the following relations:

$$
\begin{aligned}
& c_{0, e}=\delta_{0, e} \text { for }-1 \leq e \leq t-2, \\
& c_{i, 0}=\delta_{i, 0} \text { for }-1 \leq i \leq t-2, \\
& c_{i,-1}=\delta_{i,-1} \text { for }-1 \leq i \leq t-2, \\
& c_{i, r-1}=c_{i+1, r} \text { for } 0 \leq i \leq t-3 .
\end{aligned}
$$

A straightforward, but lengthy, simultaneous double induction yields that:

$$
\left\{\begin{array}{l}
c_{i, e}=0 \text { for }-1 \leq e<i \leq t-2 \\
c_{i, i}=1 \text { for }-1 \leq i \leq t-2 \\
c_{i, i+1}=0 \text { for }-1 \leq i \leq t-1
\end{array}\right.
$$

We shall next prove that

$$
c_{i, j}=\delta_{i, j} \text { for }-1 \leq i, j \leq t-2 \text { and } i \equiv 0(\bmod 2) .
$$

By (3), we know that $C\left(u_{t-2}\right)=u_{t-2}$. Suppose $C\left(u_{t-2 e+2}\right)=u_{t-2 e+2}$ where $2 \leq e \leq r$. We need to show that $C\left(u_{t-2 e}\right)=u_{t-2 e}$.

Now

$$
\begin{gathered}
C\left(u_{t-2 e+1}\right)=C\left(u_{t-2 e+2}\right) C\left(u_{-1}\right)= \\
\left(u_{t-2 e+2}\right)\left(u_{-1}+\sum_{h=1}^{t-2} c_{-1, h} u_{h}\right)= \\
u_{t-2 e+1}+\sum_{j=1}^{2 e-4} c_{-1, j} g_{j, t-2 e+2} u_{t-2 e+2+j}
\end{gathered}
$$

where $u_{m} u_{n}=g_{m, n} u_{m+n}$. (Our convention is that summations from $m$ to $n$ are taken to be zero whenever $m>n$.) Then $C\left(u_{t-2 e}\right)=C\left(u_{t-2 e+1}\right) C\left(u_{-1}\right)=$

$$
\begin{aligned}
& u_{t-2 e}+\sum_{h=1}^{2 e-3} c_{-1, h} g_{h, t-2 e+1} u_{t-2 e+1+h}+ \\
& \sum_{j=1}^{2 e-4} c_{-1, j} g_{j, t-2 e+2} u_{t-2 e+1+j}+ \\
& \sum_{h, j=1}^{2 e-5} c_{-1, h} c_{-1, j} g_{j, t-2 e+2} g_{h, t-2 e+2+j} u_{t-2 e+2+j+h}= \\
& u_{t-2 e}+c_{-1,2 e-3} g_{2 e-3, t-2 e+1} u_{t-2}+ \\
& \sum_{j=1}^{2 e-4} c_{-1, j}\left(g_{j, t-2 e+1}+g_{j, t-2 e+2}\right) u_{t-2 e+1+j}+
\end{aligned}
$$




$$
\begin{gathered}
\sum_{j=1}^{e-2}\left(c_{-1, j}\right)^{2} g_{j, t-2 e+2} g_{j, t-2 e+2+j} u_{t-2 e+2+2 j}+ \\
\sum_{h=1}^{e-3}\left[\sum _ { j = h + 1 } ^ { 2 e - 4 - h } c _ { - 1 , h } c _ { - 1 , j } \left(g_{h, t-2 e+2} g_{j, t-2 e+2+h}+\right.\right. \\
\left.\left.g_{j, t-2 e+2} g_{h, t-2 e+2+j}\right) u_{t-2 e+2+h+j}\right]
\end{gathered}
$$

It can easily be seen that for $2 \leq e \leq r$ and $1 \leq j \leq t-4$ we have both $g_{2 e-3, t-2 e+1}=0$ and $g_{j, t-2 e+1}=g_{j, t-2 e+2}$. Also, for $3 \leq e \leq r$ and $1 \leq j \leq e-2$, we have

$$
g_{j, t-2 e+2} g_{j, t-2 e+2+j}=0 .
$$

It can be shown that

$$
g_{h, t-2 e+2} g_{j, t-2 e+2+h}=g_{j, t-2 e+2} g_{h, t-2 e+2+j}
$$

for $4 \leq e \leq r, 1 \leq h \leq e-3$, and $h+1 \leq j \leq 2 e-4-h$. Therefore, $C\left(u_{t-2 e}\right)$ $=u_{t-2 e}$ and (4) is obtained.

It now follows immediately from (2) and (4) that

$$
c_{i, r-1}=\delta_{i, r-1} \text { for } 0 \leq i \leq t-3 .
$$

We now claim that:

If $B$ is defined by

$$
\begin{aligned}
& B\left(u_{i}\right)=\sum_{e=-1}^{r-2} c_{i, e} u_{e} \text { for }-1 \leq i \leq r-2, \\
& \text { then } B \in \mathscr{A}_{k} \text {. }
\end{aligned}
$$

Let $-1 \leq i, j \leq r-2$. Since $C\left(u_{i} u_{j}\right)=C\left(u_{i}\right) C\left(u_{j}\right)$, a linear independence argument yields that

$$
B\left(u_{i} u_{j}\right)=B\left(u_{i}\right) B\left(u_{j}\right)+\left(c_{i,-1} c_{j, r-1}+c_{i, r-1} c_{j,-1}\right) u_{r-2} .
$$

But by (1) and (5), we have

$$
c_{i,-1} c_{j, r-1}+c_{i, r-1} c_{j,-1}=0
$$

in all possible cases. Hence $B \in \mathscr{A}_{k}$.

A rather combinatorial, several case argument utilizing (1) and (3) proves the following partial converse to (6):

$$
\text { Let } B \in \mathscr{A}_{k} \text { with }
$$


$B\left(u_{i}\right)=\sum_{e=-1}^{r-2} b_{i, e} u_{e}$ for $-1 \leq i \leq r-2$.

If $C$ is defined by

$C\left(u_{i}\right)=\sum_{e=-1}^{r-2} b_{i, e} u_{e}$ for $-1 \leq i \leq r-2$

and

$C\left(u_{i}\right)=\sum_{e=r-1}^{t-2} b_{i-r, e-r} u_{e}$ for $r-1 \leq i \leq t-2$,

then $C \in \mathscr{A}_{k+1}$.

It now follows immediately from (6), (7), and the induction hypothesis that the matrix $\left(c_{i, j}\right)$ of an element of $\mathscr{A}_{k+1}$ has

$$
c_{i, j}=b_{i, j} \text { for }-1 \leq i, j \leq r-2
$$

where $\left(b_{i, j}\right)$ is a matrix of form $A_{k}$. Note that (3) says $c_{r+i, j}=0$ for $-1 \leq i, j \leq r-2$. That the matrix of an element of $\mathscr{A}_{k+1}$ satisfies each of the five other properties required for it to be of the form $A_{k+1}$ is reasonably direct to establish by invoking some combinatorics, linear independence, (3), and (4).

Conversely, let $A=\left(c_{i, j}\right)$ where $-1 \leq i, j \leq t-2$ be any matrix of form $A_{k+1}$. We will show that $A \in \mathscr{A}_{k+1}$. It is clear from the definition, that appropriate analogues of (3) and (4) are true for matrices of form $A_{k+1}$. Since $A$ is triangular with non-zero diagonal entries, $A$ is a non-singular linear transformation of $S\left[2^{k+1}\right]$ onto itself.

We must prove that if $u_{i} u_{j}=g_{i, j} u_{i+j}$, then

$$
g_{i, j} A\left(u_{i+j}\right)=A\left(u_{i}\right) A\left(u_{j}\right)
$$

for $-1 \leq i, j \leq t-2$. This is trivial when both $i$ and $j$ are even. When $i$ is even and $j$ is odd, it follows since

$$
g_{i, j} c_{i+j, i+n}=c_{j, n} g_{i, n}
$$

for $j+2 \leq n \leq t-2-i$.

Suppose $i$ and $j$ are both odd. Since

$$
c_{i, i+h} g_{j, i+h}=c_{j, j+h} g_{i, j+h}
$$

for $2 \leq h \leq t-2-i-j$, we have $A\left(u_{i}\right) A\left(u_{j}\right)=$

$$
g_{i, j} u_{i+j}+\sum_{m, n=2}^{t-4-i-j} c_{i, i+m} c_{j, j+n} g_{i+m, j+n} u_{i+j+m+n}
$$


If $i=j$, then $\left[A\left(u_{i}\right)\right]^{2}=$

$$
\begin{aligned}
& g_{i, i} u_{2 i}+\sum_{m=2}^{r-1-i}\left[c_{i, i+m}\right]^{2} g_{i+m, i+m} u_{2 i+2 m}+ \\
& \sum_{m, n} 2 c_{i, i+m} c_{i, i+n} g_{i+m, i+n} u_{2 i+m+n}
\end{aligned}
$$

where the last summation is taken over all $m$ and $n$ such that both $2 \leq m<n \leq t-4-2 i$ and $m+n \leq t-2-2 i$. Since $g_{h, h}=0$ for $h \neq 0$, $\left[A\left(u_{i}\right)\right]^{2}=0=A\left(u_{i}^{2}\right)$. If $i<j$, then

$$
c_{i, i+m} c_{j, j+n} g_{i+m, j+n}=0
$$

for $2 \leq m, n \leq t-4-i-j$. Hence $A\left(u_{i}\right) A\left(u_{j}\right)=g_{i, j} u_{i+j}=g_{i, j} A\left(u_{i+j}\right)$.

So in all cases, we have exhibited that a matrix of form $A_{k+1}$ is the matrix of an element of $\mathscr{A}_{k+1}$ with respect to the canonical basis. This completes our main induction and hence the proof of Theorem 1.

\section{The Automorphism groups.}

Definition. Let $\mathscr{B}_{n}$ be the group which is generated by $2^{n}-2$ generators $G_{i}$ where $1 \leq i \leq 2^{n}-2$ and $n \geq 2$. Let

$$
G_{i}^{2}=I \text { for } 1 \leq i \leq 2^{n}-2 \text {. }
$$

Define commuting relations on the generators inductively as follows: In $\mathscr{B}_{2}$, let $G_{1} G_{2}=G_{2} G_{1}$. Set $r=2^{k}$ and $s=2^{k-1}$. Assuming that $\mathscr{B}_{k}$ is defined, embed $\mathscr{B}_{k}$ as a subgroup into $\mathscr{B}_{k+1}$. Then for $1 \leq i \leq s-2$ and $-1 \leq j \leq s-2$ define

$$
G_{i} G_{r+j}=\left\{\begin{array}{l}
G_{r+1+i+j} G_{r+j} G_{i} \text { when } G_{i} G_{s+j}=G_{s+1+i+j} G_{s+j} G_{i} \\
G_{r+j} G_{i} \text { when } G_{i} G_{s+j}=G_{s+j} G_{i}
\end{array}\right.
$$

and

$$
G_{i} G_{r+s+j}=\left\{\begin{array}{l}
G_{r+s+1+i+j} G_{r+s+j} G_{i} \text { when } G_{i} G_{s+j}=G_{s+1+i+j} G_{s+j} G_{i} \\
G_{r+s+j} G_{i} \text { when } G_{i} G_{s+j}=G_{s+j} G_{i} .
\end{array}\right.
$$

Also for $-1 \leq i, j \leq s-2$ define

$$
G_{s+i} G_{r+j}=\left\{\begin{array}{l}
G_{r+s+1+i+j} G_{r+j} G_{s+i} \text { when } i+j \text { is odd and } i+j<s-2 \\
G_{r+j} G_{s+i} \text { otherwise }
\end{array}\right.
$$

and

$$
G_{s+i} G_{r+s+j}=G_{r+s+j} G_{s+i}
$$


Finally for $-1 \leq i<j \leq r-2$ define

$$
G_{r+i} G_{r+j}=G_{r+j} G_{r+i} \text {. }
$$

THEOREM 2. The automorphism group of the Jordan algebra $S\left[2^{n}\right]$ over the field $J_{2}$ is $\mathscr{B}_{n}$; that is, the set of all matrices of the form $A_{n}$ forms a group under multiplication and is $\mathscr{B}_{n}$.

Proof. The proof is by induction on $n$. Since it represents all automorphisms of $S\left[2^{n}\right]$ by Theorem 1 , the set of all matrices of the form $A_{n}$ forms a group $\mathscr{A}_{n}$ under multiplication. Clearly, the order of $\mathscr{A}_{n}$ is $2^{2^{n}-2}$.

Let $\left[i_{1}, i_{2}, \cdots, i_{m}\right]$ denote that element of $\mathscr{A}_{n}$ with

$$
a_{i_{j}}= \begin{cases}1 & \text { for } 1 \leq j \leq m \\ 0 & \text { otherwise }\end{cases}
$$

where $i_{1}<i_{2}<\cdots<i_{m}$ and $1 \leq m \leq 2^{n}-2$.

We begin by considering $\mathscr{A}_{2}$. Let $G_{1}=[1]$ and $G_{2}=[2]$. Then $G_{1}{ }^{2}=G_{2}{ }^{2}=I$ and $G_{1} G_{2}=[1,2]=G_{2} G_{1}$. So $G_{1}$ and $G_{2}$ generate $\mathscr{A}_{2}$. Hence $\mathscr{A}_{2}$ is $\mathscr{B}_{2}$.

Observe that $\mathscr{A}_{k}$ may be considered as a subgroup of $\mathscr{A}_{k+1}$ by identifying $\left[i_{1}, i_{2}, \cdots, i_{m}\right] \in \mathscr{A}_{k}$ with $\left[i_{1}, i_{2}, \cdots, i_{m}\right] \in \mathscr{A}_{k+1}$ where $1 \leq m \leq r-2$.

Let $G_{i}=[i]$. Suppose that $\mathscr{A}_{k}$ is $\mathscr{B}_{k}$ for this choice of the $G_{i}$. We will show that these $G_{i}$ satisfy the defining relations of $\mathscr{B}_{k+1}$ and generate $\mathscr{B}_{k+1}$.

Suppose $-1 \leq i, j \leq s-2$. By the definition of matrices of the form $A_{k+1}$, we can write

$$
G_{s+i}=\left(\begin{array}{cccc}
1 & X & 0 & 0 \\
0 & 1 & 0 & 0 \\
0 & 0 & 1 & X \\
0 & 0 & 0 & 1
\end{array}\right) \text { and } G_{r+j}=\left(\begin{array}{cccc}
1 & 0 & Y & 0 \\
0 & 1 & 0 & Y \\
0 & 0 & 1 & 0 \\
0 & 0 & 0 & 1
\end{array}\right)
$$

where $X$ and $Y$ are certain $s$ by $s$ matrices and 0 and 1 are the $s$ by $s$ zero and identity matrices respectively. Let $H \in \mathscr{A}_{k+1}$ with

$$
G_{s+i} G_{r+j}=H G_{r+j} G_{s+i} \text {. }
$$

Then 


$$
H=\left(\begin{array}{llll}
1 & 0 & 0 & Z \\
0 & 1 & 0 & 0 \\
0 & 0 & 1 & 0 \\
0 & 0 & 0 & 1
\end{array}\right)
$$

where $Z=X Y-Y X$. It can be shown that

$$
\left(\begin{array}{cccc}
1 & 0 & 0 & X Y \\
0 & 1 & 0 & 0 \\
0 & 0 & 1 & 0 \\
0 & 0 & 0 & 1
\end{array}\right)
$$

is $G_{r+s+1+i+j}$ when $i$ is odd and $i+j<s-2$ and is $I$ otherwise. It follows that

$$
H= \begin{cases}G_{r+s+1+i+j} & \text { when } i+j \text { is odd and } i+j<s-2 \\ I & \text { otherwise. }\end{cases}
$$

The other commuting relations follow by similar block matrix arguments. Using the induction hypothesis it is easy to verify that $G_{i}{ }^{2}=I$ for all $i$.

We must still show that the set $\left\{G_{i}: 1 \leq i \leq t-2\right\}$ generates $\mathscr{A}_{k+1}$. We will exhibit an algorithm for obtaining an element $\left[i_{1}, i_{2}, \cdots, i_{m}\right]$ of $\mathscr{A}_{k+1}$, where $1 \leq m \leq t-2$, as a product of the $G_{i}$. This will be done by induction on $m$. For $m=1,\left[i_{1}\right]=G_{i_{1}}$.

Suppose that $\left[i_{1}, i_{2}, \cdots, i_{m-1}\right]$ has been expressed as a product of the $G_{i}$. We will express $\left[i_{1}, i_{2}, \cdots, i_{m-1}, i_{m}\right]$ as a product of $\left[i_{1}, i_{2}, \cdots, i_{m-1}\right]$ and the $G_{i}$.

Now $G_{e}=[e]=\left(c_{i, j}\right)$, where $1 \leq e \leq t-2$, has the properties that

$$
\left\{\begin{array}{l}
c_{i, j}=\delta_{i, j} \text { for }-1 \leq i \leq e,-1 \leq j \leq e-1 \\
c_{-1, e}=1 \\
c_{i, e}=\delta_{i, e} \text { for } 0 \leq i \leq e .
\end{array}\right.
$$

Hence

$$
\begin{aligned}
& {\left[i_{1}, i_{2}, \cdots, i_{m-1}\right] G_{i_{m}}=} \\
& {\left[i_{1}, i_{2}, \cdots, i_{m-1}, i_{m}, j_{1,1}, j_{1,2}, \cdots, j_{1, m_{1}}\right]}
\end{aligned}
$$


where $j_{1,1}>i_{m}$ and $0 \leq m_{1} \leq t-2-i_{m}$. If $m_{1}=0$, then we are done. If not, then

$$
\begin{aligned}
& {\left[i_{1}, i_{2}, \cdots, i_{m-1}\right] G_{i_{m}} G_{j_{1,1}}=} \\
& {\left[i_{1}, i_{2}, \cdots, i_{m-1}, i_{m}, j_{2,1}, j_{2,2}, \cdots, j_{2, m_{2}}\right]}
\end{aligned}
$$

where $j_{2,1}>j_{1,1}$ and $0 \leq m_{2} \leq t-2-j_{1,1}$. If $m_{2}=0$, then we are done. If not, then

$$
\begin{aligned}
& {\left[i_{1}, i_{2}, \cdots, i_{m-1}\right] G_{i_{m}} G_{j_{1,1}} G_{j_{2,1}}=} \\
& {\left[i_{1}, i_{2}, \cdots, i_{m-1}, i_{m}, j_{3,1}, j_{3,2}, \cdots, j_{3, m_{3}}\right]}
\end{aligned}
$$

where $j_{3,1}>j_{2,1}$ and $0 \leq m_{3} \leq t-2-j_{2,1}$. If $m_{3}=0$, then we are done. If not, then consider

$$
\left[i_{1}, i_{2}, \cdots, i_{m-1}\right] G_{i_{m}} G_{j_{1,1}} G_{j_{2,1}} G_{j_{3,1}} \text {. }
$$

It is clear that this process must terminate. This completes the proof of Theorem 2.

\section{REFERENCE}

[1] E.C. Paige, Jr: Jordan Algebras of Characteristic Two, Dissertation, University of Chicago, Chicago, Illinois, (1954).

Georgetown University 